\title{
Germanica
}

\section{Die « neue Sprache » bei Christa Wolf : Utopie und Wirklichkeit}

\section{Gunhild Samson}

\section{(2) OpenEdition \\ 1 Journals}

Édition électronique

URL : http://journals.openedition.org/germanica/2342

DOI : 10.4000/germanica.2342

ISSN : 2107-0784

Éditeur

Université de Lille

\section{Édition imprimée}

Date de publication : 30 décembre 1999

ISBN : 9782913857018

ISSN : 0984-2632

\section{Référence électronique}

Gunhild Samson, «Die « neue Sprache » bei Christa Wolf : Utopie und Wirklichkeit », Germanica [En ligne], 25 | 1999, mis en ligne le 30 janvier 2014, consulté le 06 octobre 2020. URL : http:// journals.openedition.org/germanica/2342; DOI : https://doi.org/10.4000/germanica.2342

Ce document a été généré automatiquement le 6 octobre 2020.

(c) Tous droits réservés 


\title{
Die « neue Sprache » bei Christa Wolf : Utopie und Wirklichkeit
}

\author{
Gunhild Samson
}

1 Christa Wolfs poetisches wie essayistisches Werk durchzieht wie ein Leitfaden, über die Motive der Unmöglichkeit des Sprechens und der Suche nach einer neuen Sprache, die Auseinandersetzung des Individuums mit dem herrschenden Denken und Sprechen. Das «Ich », das sich durch das «Ich-Sagen » selbst finden und in der öffentlichen Sprache einbringen will, scheitert jeweils an dem die Gesellschaft beherrschenden Diskurs und verstummt, sei es durch Selbstzensur, sei es im Tod. Doch steht am Horizont die Utopie einer neuen, wiedergefundenen Sprache, die sich im Gespräch realisiert.

2 Ich möchte im Folgenden diesem Spannungsverhältnis zwischen individueller Sprache und herrschendem Diskurs in zwei Prosatexten und einer öffentlichen Rede nachgehen.

\section{Kommunikationssituation und Wahl der Textsorten}

Die Erzählungen Was bleibt ${ }^{1}, 1979$ entstanden, aber im Herbst 1989 überarbeitet und 1990 erschienen, sowie Kassandra 2,1983 erschienen, und die Rede Sprache der Wende vom 4. November $1989^{3}$ weisen zeitliche, textuelle, intertextuelle und kommunikativpragmatische Bezüge auf. Die beiden Prosatexte entstanden in einer Kommunikationssituation, die vom Monopol der offiziellen Partei- und Staatssprache geprägt war und in der Sprachkritik als Form der Gesellschaftskritik nur in impliziter, verschlüsselter Form möglich war. Offene Kritik hatte Ausgrenzung oder Ausbürgerung zur Folge. Die Rede Sprache der Wende dagegen wurde in einer Zeit des gesellschaftlichen Umbruchs gehalten, in der die Staatsmacht ins Wanken geraten war und alle Hoffnungen auf Mitsprache und demokratische Umgestaltung erlaubt waren. Auch das Verhältnis zwischen der Autorin und den Adressaten ihrer Schriften sowie die zur Verfügung stehenden Textsorten, hatten sich von Grund auf verändert : Was bleibt mußte von Anfang an wegen seiner thematischen Brisanz für die Schublade bzw. für einen hypothetischen späteren Leser konzipiert werden, also ohne wirkliche Bindung 
an den Leser. Die explizite Art der Behandlung des Themas der Zerstörung des Individuums durch das Vorgehen des Staatssicherheitsdienstes erforderte 1979 die Wahl der Textsorte "Tagebuchaufzeichnung $»^{4}$. Die ständige Beschwörung der neuen Sprache macht den Bezug zum Zeitpunkt der Überarbeitung im Herbst 1989 offenbar, läßt diesen aber auch als ein hypothetisches « eines Tages » im Ungewissen.

Die Erzählung Kassandra, in der dargestellt wird, wie sich der herrschende Diskurs mit Hilfe des Polizeiapparates konstituiert und die Öffentlichkeit manipuliert, konnte durch die Versetzung des Stoffes ins Mythische und die implizite, metaphernartige Erzählweise der Zensur entgehen. Kassandra, die Seherin, hatte die Botschaft zu überbringen, daß «Troia untergehen werde » Diese wurde, wie Christa Wolf es 1989 selbst äußerte ${ }^{5}$, vom aufmerksamen Leser in der DDR sehr wohl verstanden.

Mit der Rede vom 4. November 1989 Sprache der Wende trat Christa Wolfs zum ersten Mal an die Öffentlichkeit, nachdem sie ab 1965, nach ihrer Rede auf dem 11. Plenum des ZK der SED, in eine Außenseiterrolle gedrängt wurde und ihr seitdem der Zugang zu den Medien verwehrt war. Das Wunschbild der neuen, befreiten Sprache, gegründet auf einem solidarischen Verhältnis zwischen den Sprechern, scheint während der revolutionären Bewegung eine kurze Zeit lang Wirklichkeit geworden zu sein. Christa Wolf formuliert dies mit dem Bild der befreiten Sprache und führt modellhaft in ihrer Rede die Möglichkeit der Realisierung dieser neuen Sprache vor.

\section{Kritik am herrschenden Diskurs in Was bleibt und Kassandra}

6 Was bleibt, eine Erzählung, in der Christa Wolf in autobiographischen Zügen ${ }^{6}$ den Tagesablauf einer von der Staatssicherheit bespitzelten Schriftstellerin beschreibt, beginnt mit der Beschwörung der neuen Sprache : «Nur keine Angst. In jener anderen Sprache, die ich im Ohr, noch nicht auf der Zunge habe, werde ich eines Tages darüber reden. Heute, das wußte ich, wäre es noch zu früh. Aber würde ich spüren, wenn es an der Zeit ist? Würde ich meine Sprache wiederfinden? » (Wb 7). Und als würde sich ein Kreis $^{7}$ schließen, endet die Erzählung mit der gleichen Heraufbeschwörung der jetzt noch als Wunschtraum gesehenen neuen Sprache, mit der gleichen Hoffnung auf deren Realisierung. Der Zeitpunkt dafür ist unbekannt, erscheint nur als ein ungewisses, aber erhofftes « eines Tages» : «Diesmal haben sie [...] den Punkt getroffen. Den ich eines Tages, in meiner neuen Sprache, benennen würde. Eines Tages, dachte ich, werde ich sprechen können, ganz leicht und frei. Es ist noch zu früh, aber es ist nicht immer zu früh. » (Wb 107). Da die neue Sprache, « die härter sein würde » (Wb 10), d.h. in der sie Widerstand leisten könnte, ihr versagt ist, bleibt als einzige reale Möglichkeit das einfache Aufschreiben dessen « Was bleibt. Was meiner Stadt zugrunde liegt und woran sie zugrunde geht. » (Wb 107/108). Und die Erzählerin kehrt an den Anfang des Tages zurück, um in der alten Sprache, « in der sie noch denken mußte » (Wb 10), « darüber » zu reden, d.h. Zeugnis abzulegen und den durch die Bespitzelung hervorgerufenen Zustand der Lähmung, der ständigen Angst, der Einschüchterung möglichst genau zu beschreiben.

7 Ziel der immer wiederkehrenden Beschwörung der neuen Sprache ist es, auf ihrem Hintergrund die Unzulänglichkeit der alten Sprache sprachkritisch aufzudecken und den herrschenden Diskurs zu denunzieren. Da lexikalische und thematische Ähnlichkeiten 
in der Art der Kritik bestehen, sollen die Merkmale dieses Diskurses im Folgenden an Hand beider Erzählungen zusammenfassend dargestellt werden :

- Die Allmacht und Allgegenwart, aber zugleich Anonymität des Diskurses wird denunziert : «er, der eigentliche Meister, der wirkliche Herr » (Wb 53), « der Meister, der die Stadt beherrschte " (Wb 34), mit seinen «Gehilfen » (Wb 34), « die jungen Herren» (Wb 28; 51); die "Machtvollkommenheit» des Eumelos (K 305), die allgegenwärtigen « jungen Männer » der Palastwache (K 256-257).

9 - Die Verwendung einer spezifischen Lexik, welche von der Alltagssprache abweicht und die Praxis des unmenschlichen Polizei- und Überwachungsstaates widerspiegelt, wird entlarvt: "Verhör» (Wb 66), «mit der Polizei auseinandertreiben» die «Zusammenrottung sei ausfallend und aggressiv », die «Zusammenrottung auflösen », «Polizeieinsatz», «Einsatzwagen» «Hausfriedensbruch» (Wb 99/ 100); das "Sicherheitsnetz », in das jeder gerät, der sich dem nähert, der, der "Konspiration verdächtig », " unter Beobachtung gestellt » ist (K 275) und bald über ganz Troia ausgebreitet wird : « es betraf nun jedermann » (K 305), « strenge Kontrollen » werden durchgeführt "Sonderbefugnisse für die Kontrollorgane " gelten, "Maßnahmen » werden ergriffen, für die überdies « Unterstützung » gefordert wird (K 305).

10 - Die Sprachregelung als Einführung neuer Wörter und Tabuisierung alter Wörter wird aufgedeckt. Sehr deutlich wird beschrieben, wie der herrschende Diskurs sich durch Sprachregelung und Sprachmanipulierung konstituiert und verfestigt: Eumelos entscheidet über den " Gebrauch von Wörtern » (K 256) und beargwöhnt jeden, der sich dem neuen Gebrauch nicht unterwirft, der seit Anfang des Krieges aufgezwungen wird : «Krieg durfte er nicht heißen. Die Sprachregelung lautete, zutreffend : Überfall. (K 273); "Er habe sie opfern müssen, aber "morden" und "schlachten" sind ja den Mördern und Schlächtern unbekannt » (K 254).

11 -Es wird bloßgestellt, welche Rolle das vereinnahmende "wir» bei der Sprachregelung spielt, z.B. in Priamos Antwort auf die Frage Kassandras, warum sie Krieg um ein Phantom führen : "Wir, denk ich, werden angegriffen werde, wir denk ich, setzen uns zur Wehr » (K 272, auch 273/297).

12 - Sprachlosigkeit wird als Folge der sich in Wortschwall auflösenden Phrasen erkannt. Diese im oppositionellen Diskurs vom Herbst $1989^{8}$ weithin intertextuell gebrauchte Metapher, die die "gestörte Kommunikation zwischen Staat und Gesellschaft $»^{9}$ veranschaulicht, dient bei Christa Wolf einerseits als Bezeichnung für die sprachlose Macht und deren Helfer, die durch Lügen sprachlos wird, andererseits für den sprachlosen Zustand des Individuums, das zum Schweigen gezwungen wurde. Morde, auf denen die Macht gegründet ist, werden verheimlicht (K 254/255). Die «jungen Herren" haben einen "gläsernen Blick» (Wb 51), da sie trotz aller Beobachtung nicht mehr « sehen », d.h. « sprechen » können, denn Lügen pervertieren den Lügner und führen zur "Sprachlosigkeit von Gewissenlosen.» - "Meine neue Sprache... müßte auch von ihnen sprechen können, wie sie sich jeglicher Sprachohnmacht annehmen sollte. (Wb 30-31).

13 - Die sprachlichen und moralischen Folgen für die vom herrschenden Diskurs Unterdrückten werden schonungslos aufgedeckt. Es sind Verurteilung zu Sprachlosigkeit und Schweigen oder Pervertierung zu Anpassung und Lüge. Kassandra, die sich als Seherin zum Sprechen ausersehen hatte, deren Prophezeiungen aber nicht gehört werden, entlarvt den Krieg als «Sprachkrieg» (K 267), unterwirft sich der Sprachregelung nicht und verstummt. Sie geht sprachlos in den Tod : «Das Letzte wird 
ein Bild sein, kein Wort. Vor den Bildern sterben die Worte. Todesangst. » (K 221). In Kassandra wie in Was bleibt steht die resignierte Frage am Ende, ob und wann, nach welch innerem Konflikt der Wunschtraum der anderen Sprache sich erfüllen kann: «Ich will nicht mehr sprechen. [...] Wer wird, und wann, die Sprache wiederfinden. Einer, dem ein Schmerz den Schädel spaltet, wird es sein. » (K 206).

Dieses Bild des äußersten Konfliktes im Bewußtsein veranschaulicht eines der großen intertextuellen Themen von Christa Wolf: das Verhältnis von Sprache und Moral: «Wer aber sagte uns, was Würde sei ?» (Wb 53). Wie soll, wie kann sich der Mensch in einem totalitären System, das ihn zur Lüge zwingt, verhalten? Wie kann er Würde bewahren? Ist Widerstand möglich ? Ohne Beschönigung stellt Christa Wolf dar, wie allgegenwärtige Machtstrukturen zur Entwürdigung, zur Selbstaufgabe durch Anpassung führen : « Und bis dahin, bis hin zu ihm, nur das Gebrüll und der Befehl und das Gewinsel und das Jawohl der Gehorchenden" (K 206) und beschreibt den Deformationsprozeß, den Menschen durchmachen, die ständig der Angst ausgesetzt sind : "Worauf sollten sie setzen : auf meinen Hang zur Übereinstimmung mit den Herrschenden oder auf meine Gier nach Erkenntnis. » (K 264). Kassandra erschrickt über sich selbst, daß sie mit Eumelos, dem Vertreter der Macht und Organisator des Überwachungsdienstes, « in einen inneren Dialog verstrickt » sei (K 306), dieser innere Dialog, der auch als «innerer Begleiter», als Dialog mit dem "Dritten», der " unwillkommenen Stimme»das Denken und Sprechen der Erzählerin in Was bleibt beherrscht (Wb 56ff.). Rückblickend äußert sich Christa Wolf im Oktober 1989 über diese Verführung durch die Macht und die daraus resultierenden Deformationen : « Das war eine der schlimmsten Entwicklungen bei uns durch diese rigiden Machtstrukturen, daß man sich herausziehen mußte aus den Apparaten, um nicht von ihnen deformiert zu werden ${ }^{10}$. $\gg$ Christa Wolfs Verdienst ist es, diese Zerstörungskraft und Verführung an den beiden Frauenfiguren aufgedeckt zu haben, an der Figur Kassandras wie auch an der Figur der Erzählerin in Was bleibt, die im Kreislauf der Angst, Selbstaufgabe und kompromißlosen Anpassung verstrickt bleibt und deren kommunikative Beziehungen zersetzt sind. Da die Erzählerin sich weder zu einer neuen, härteren Sprache durchringen noch der Macht widerstehen kann, die sie in ihrem Schreiben lähmt, pervertiert ihre Sprache zu belanglosen Stereotypen.

\section{Utopie und Realität der neuen Sprache}

In der düsteren, von Bespitzelung und Entwürdigung überschatteten Atmosphäre der beiden Erzählungen leuchtet hin und wieder die Möglichkeit der neuen Sprache auf. Diese erscheint in Kassandra als eine außerhalb der Gesellschaft zu realisierende Utopie und in Was bleibt als mögliche, aber der Gefahr der Verfolgung ausgesetzte Widerstandshandlung gegen den auferlegten Diskurs. Diese neue Sprache kann sich nur im Gespräch ${ }^{11}$ realisieren und wird häufig mit den Adjektiven « leicht und frei » (Wb 10), « heiter » und « locker » (Wb 97) gekennzeichnet; sie ist « wahr » (Wb 76), der Sprecher ist nicht « erpreßbar », er kehrt dabei sein « innerstes Wesen » hervor (Wb 77), er redet « in zutraulichem Zustand », wie es bei dem jungen Mädchen in Was bleibt der Fall ist. Oder, wie nach dem pervertierten Leseabend, also außerhalb des verordneten Diskurses, wo das Wort «Brüderlichkeit » wie ein «utopisches Wort » fällt, "die wirklichen Fragen » behandelt werden und « jeder sich selbst aussprach » (Wb 96). 
16 Was hier als die neue brüderliche Sprache dargestellt wird, erinnert an die Utopie des zwanglosen Miteinandersprechens bei den Frauen auf dem Ida-Berg in Kassandra : «Ich sagte und war selbst verwundert, wie natürlich meine Stimme klang [...] Da entstand ein Schweigen, in das meine Stimme paßte ; nun hatte sie genau den Raum gefunden, der für sie vorgesehen war. » - "Wir sprachen rückhaltlos, freundlich und sachlich.» (K 327).

17 Als Christa Wolf am 4. November 1989, auf der Großdemonstration für Meinungs-, Rede- und Pressefreiheit auf dem Alexanderplatz in Berlin, ihre Rede über die Sprache der Wende mit den Worten beginnt: «Jede revolutionäre Bewegung befreit auch die Sprache. Was bisher so schwer auszusprechen war, geht uns auf einmal frei über die Lippen " (D 119), nimmt sie ihre eigenen und die in verschiedenen Varianten im Oppositionsdiskurs gängigen Metaphern von der angeketteten Sprache, der Sprachlosigkeit, der Befreiung der Sprache wieder auf und schreibt sich damit intradiskursiv in den Oppositionsdiskurs ein, der erst seit kurzer Zeit in die Öffentlichkeit gedrungen war. Wie bewußt sie den Herbst 1989 als eine Zeit der neuen Sprache gelebt hat und auch heute noch so versteht, geht aus ihrer letzten Textsammlung Hierzulande Andernorts ${ }^{12}$ hervor: « ...und wir holten uns wieder, was uns aus den Händen genommen war : Das Recht, frei unsere kritische Meinung zu äußern. Die Verhältnisse beim Namen zu nennen ${ }^{13}$. »

18 Wie Christa Wolf das Wirken der neuen Sprache im öffentlichen Diskurs versteht, bezeugen Kommunikationsstil und Thematik dieser Rede. Deren Merkmale sollen hier kurz erläutert werden.

19 -Adressatenbeziehung:

20 Der Abdruck ihres Redebeitrages in Im Dialog beginnt ohne Anrede. Damit setzt sie ein Signal, wie ihre Sprecherrolle verstanden werden soll ${ }^{14}$ und nimmt bewußt Abstand von den alten staatlichen Verlautbarungen (z.B. noch die Rede von Egon Krenz am 18. Oktober 1989). Sie verweigert die asymmetrische Beziehung von oben nach unten, die sich als Verkündigung einer Wahrheit versteht. Da der Dialog ${ }^{15}$ als Gespräch zwischen gleichberechtigten Partnern für Christa Wolf die einzig wahre Form des Meinungsaustausches ist, gestaltet sie ihre Rede in der Form eines "öffentlichen Selbstgesprächs $»^{16}$ mit den von ihr gemeinten Adressaten, seien es Demonstranten, Mitläufer oder Anhänger der Staatsmacht.

21 - Äußerst häufige Verwendung des integrierenden Pronomens « wir "und « uns » :

22 Im Gegensatz $\mathrm{zu}$ der Funktion des vereinnahmenden «wir» des herrschenden Diskurses hat das "wir » hier die Funktion, eine Gemeinschaft auf solidarischer Basis zu stiften. Christa Wolf schafft mit dem "wir» eine Gefühlslage, in der sie als Redesubjekt in der "wir »-Gruppe aufgeht und so mit ihr ein kollektives Redesubjekt

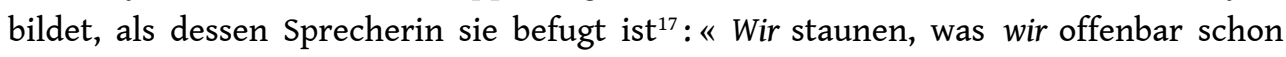
lange gedacht haben und was wir uns jetzt laut zurufen... wir erinnern uns...». Diese "wir-Gruppe » umfaßt alle, die sich von den ihr ausgesprochenen Hoffnungen und Wünschen betroffen fühlen: die oppositionellen Gruppen, die Demonstranten und das Volk.

23 - Verwendung der « ich »-Form und metasprachliche Bezeichnungen :

24 Mit der « ich »-Form zeigt Christa Wolf, daß das «Ich » sich jetzt selbst setzen und zu sich selbst finden kann, indem es eine eigene Meinung äußert, sei es um sich kritisch von Ausdrücken abzusetzen, die schon von der Staatsmacht vereinnahmt werden: 
"Mit dem Wort "Wende" habe ich da meine Schwierigkeiten. Ich sehe da ein Segelboot, der Kapitän ruft...", sei es um eigene Vorschläge einzubringen: "Ich würde von revolutionärer Erneuerung sprechen », oder auch, um die eigene Betroffenheit offenbar zu machen : «Und dies ist für mich der wichtigste Satz dieser letzten Wochen - der tausendfache Ruf : Wir - sind - das Volk !» der Suche nach der neuen Sprache. Diese authentische Sprache, die in den Erzählungen Was bleibt und Kassandra nur als Utopie oder einzelne Widerstandshandlung am Rande der Gesellschaft realisiert werden kann, da der offizielle Diskurs die Öffentlichkeit durch Gewalt beherrscht und das Individuum pervertiert, ist für Christa Wolf in der kurzen Zeit der revolutionären Erneuerung im Herbst 1989 Wirklichkeit geworden. Die Rede vom 4. November ist ein gelungenes Beispiel dafür, wie das Ich sich in der neuen 
Sprache selbst setzen kann. Christa Wolf lebt und spricht dies vor, indem sie sich nicht als allmächtiges Redesubjekt betrachtet, sondern im kollektiven Redesubjekt aufgeht, das sich in der Rede und durch die Rede, während des Sprechens selbst konstituiert. Alle verwendeten rhetorischen Mittel dienen dazu, am diskursiven Vorgang selbst aufzuzeigen, daß es möglich ist, die neue Sprache zu realisieren.

\section{NOTES}

1. Christa Wolf, Was bleibt, Frankfurt, Luchterhand 1990 (zitiert Wb).

2. Christa Wolf, Kassandra. Vier Vorlesungen. Eine Erzählung, Berlin, Aufbau Verlag 1983 (zitiert K).

3. Christa Wolf, «Sprache der Wende, Rede auf dem Alexanderplatz, 4. November 1989 ». In : Christa Wolf, Im Dialog, Aufbau-Verlag 1990 ; Luchterhand 1993, S. 119-121 (zitiert D); auch in Christa Wolf, Auf dem Weg nach Tabou. Texte 1990-1994, Köln, Kiepenheuer u. Witsch 1994, S. 11-13 (zitiert T).

4. Ich gehe hier nicht auf die Kontroverse ein, die das Erscheinen des Buches 1990 bei westdeutschen Intellektuellen auslöste. Einer der Gründe dafür mag auch die Textsortenwahl gewesen sein.

5. Christa Wolf, Brief an Günter Grass vom 21.3.1993 : «Ich habe dieses Land geliebt. Daß es am Ende war, wußte ich, weil es die meisten Leute nicht mehr integrieren konnte, weil es Menschenopfer forderte. Ich habe das in "Kassandra » beschrieben, die Zensur stocherte in den "Vorlesungen» herum; ich wartete gespannt, ob sie es wagen würden, die Botschaft der Erzählung zu verstehen, nämlich daß Troja untergehen muß. Sie haben es nicht gewagt und die Erzählung ungekürzt gedruckt. Die Leser in der DDR verstanden sie. In: Akteneinsicht Christa Wolf. Zerrspiegel und Dialog. Eine Dokumentation. Hg. von Hermann Vinke, Luchterhand 1993, S. 308.

6. Akteneinsicht Christa Wolf., a.a.O., S. 265 ff. Gegen Christa Wolf und ihren Mann Gerhard Wolf wurde am 12.2.1969 ein «operativer Vorgang" mit dem Kennzeichen «Doppelzüngler» angelegt. Beide waren bis 1989 wegen « staatsfeindlicher Tätigkeit » unter ständige Beobachtung gestellt.

7. Zur Ringstruktur vgl. Arker, Dieter (1994) : « Anmerkungen zu 'Was bleibt' » In : Text+Kritik. 46, hg. von Heinz Ludwig Arnold, S. 92.

8. Vgl. Samson, Gunhild (1994) : « Schlüsselwörter der Wende : Sprachlosigkeit und Dialog. » In : Heringer/Samson/Kauffmann u.a. (Hg.), Tendenzen der deutschen Gegenwartssprache, Asnières, PIA ; Tübingen, Niemeyer. S.191-212.

9. «In unserem Lande ist die Kommunikation zwischen Staat und Gesellschaft offensichtlich gestört. » Gründungsaufruf des Neuen Forum. In : Schüddekopf, Charles (Hg.) (1990) : «Wir sind das Volk! Flugschriften, Aufrufe und Texte einer deutschen Revolution, Reinbek, Rowohlt. S. 29.

10. « Leben oder gelebt werden. Gespräch mit A. Nehring, 30. Oktober 1989 ». In : Im Dialog, a.a.O. S. 112.

11. In «Subjektive Authentizität », Gespräch mit Hans Kaufmann, zitiert Christa Wolf Erasmus von Rotterdam : »Alles, was ich sage, sei Gespräch. Nichts davon sei ein Rat ». In : Christa Wolf, Die Dimension des Autors, Berlin, Aufbau-Verlag 1986, Bd. II, S.323.

12. «Daß er [F. Fühmann] den Herbst 89 hätte miterleben und mitgestalten können - das hätte ich ihm dringlich gewünscht... Es zeigte sich, der Funken, den wir manchmal für erloschen hielten, war doch nicht ganz ausgetreten worden, auf einmal kam, wenn auch... nur für kurze 
Zeit, die kreative Seite der Widersprüche, doch noch richtig hervor... und gab den Menschen, die wir zu kennen glaubten, veränderte Gesichter, neuen Mut, eine neue Sprache. » - « Nirgend sein o Nirgends du mein Land. » In : Hierzulande. Andernorts, München, Luchterhand, 1999, S. 67.

13. «Dünn ist die Decke der Zivilisation - Musikalische Meditation. Joseph Haydn, Missa in Tempore Belli. In : Hierzulande. Andernorts, München, Luchterhand, 1999, S. 209-210.

14. Volmert, Johannes (1992) : "Auf der Suche nach einer neuen Rhetorik. Ansprachen auf den Massendemonstrationen Anfang November 1989.» In: Burkhardt, Fritzsche (Hg.), Sprache im Umbruch. Politischer Sprachwandel im Zeichen von "Wende » und "Vereinigung ", Berlin, New York: de Gruyter, S. 80.

15. Aufschlußreich ist auch, daß der zuerst im Aufbau-Verlag erschienene Band Reden im Herbst im Neudruck bei Luchterhand unter dem Titel Im Dialog erschien.

16. Hopfer, Reinhard (1992) : «Christa Wolfs Streit mit dem 'großen Bruder'. Politische Diskurse der DDR im Herbst 1989 ». In : Burkhardt A./Fritzsche K.P. (Hg.) : Sprache im Umbruch. Politischer Sprachwandel im Zeichen von "Wende » und «Vereinigung », Berlin, New York : de Gruyter S.111-131. 17. Hopfer, Reinhard, a. a. O. S. 121.

18. Stefan Heym in seiner Rede vom 4. November : "Wir haben in diesen letzten Wochen unsere Sprachlosigkeit überwunden und sind jetzt dabei, den aufrechten Gang zu lernen.» In: Schüddekopf C., a.a.O., S. 207.

19. Christa Wolf, Medea Stimmen, Luchterhand, 1966.

\section{AUTEUR}

\section{GUNHILD SAMSON}

Université de Paris III 